\title{
A 4-channel 3 Tesla phased array receive coil for awake rhesus monkey fMRI and diffusion MRI experiments
}

\author{
Mark Haig Khachaturian ${ }^{1,2}$ \\ ${ }^{1}$ Athinoula A. Martinos Center for Biomedical Imaging, Massachusetts General Hospital, Harvard Medical School, Charlestown, \\ USA; \\ ${ }^{2}$ Department of Nuclear Science and Engineering, Massachusetts Institute of Technology, Cambridge, USA. \\ Email: markk@nmr.mgh.harvard.edu
}

Received 6 October 2010; revised 18 October 2010; accepted 20 October 2010.

\begin{abstract}
Awake monkey fMRI and diffusion MRI combined with conventional neuroscience techniques has the potential to study the structural and functional neural network. The majority of monkey fMRI and diffusion MRI experiments are performed with single coils which suffer from severe EPI distortions which limit resolution. By constructing phased array coils for monkey MRI studies, gains in SNR and anatomical accuracy (i.e., reduction of EPI distortions) can be achieved using parallel imaging. The major challenges associated with constructing phased array coils for monkeys are the variation in head size and space constraints. Here, we apply phased array technology to a 4-channel phased array coil capable of improving the resolution and image quality of full brain awake monkey fMRI and diffusion MRI experiments. The phased array coil is that can adapt to different rhesus monkey head sizes (ages 4-8) and fits in the limited space provided by monkey stereotactic equipment and provides SNR gains in primary visual cortex and anatomical accuracy in conjunction with parallel imaging and improves resolution in fMRI experiments by a factor of $2(1.25 \mathrm{~mm}$ to $1.0 \mathrm{~mm}$ isotropic) and diffusion MRI experiments by a factor of $4(1.5 \mathrm{~mm}$ to $0.9 \mathrm{~mm}$ isotropic).
\end{abstract}

Keywords: 4-Channel; fMRI; RF Coil Design; Monkey; SNR; G-Factor; Diffusion MRI; Phased Array

\section{INTRODUCTION}

Awake monkey fMRI combined with conventional neuroscience techniques (e.g. electrophysiology, lesion studies, reversible deactivation) has to potential to understand and map the functional network [1-5]. Also, awake monkey fMRI can be used to validate diffusion tracto- graphy methods [6-9] by benchmarking known functional pathways. The majority of monkey MRI experiments are performed with single coils $[1,3,4]$. Though previous monkey studies have provided valuable information, single coil full brain fMRI studies suffer from severe EPI distortions at resolutions higher than $1.25 \mathrm{~mm}$ isotropic for fMRI $[1,3,4]$.

DTI studies at $1.5 \mathrm{~mm}$ isotropic have provided valuable information on the the underlying structure of the monkey brain [10], however considering the thickness of white matter in the optic radiation is $1 \mathrm{~mm}$, current DTI studies do not possess the necessary resolution to characterize the white matter structure throughout the brain. Since the purpose of DTI is to measure the "structural connectivity" between regions of the brain, resolutions of $<1$ $\mathrm{mm}$ would be desirable. Also, more advanced diffusion reconstruction techniques which are aimed at resolving multiple fiber orientations can benefit from any SNR and EPI distortion reduction [11-13]. Phased array coils in conjunction with parallel imaging could provide gains in SNR and anatomical accuracy (i.e., reduction of EPI distortions) in order to achieve the resolutions of $1 \mathrm{~mm}$ [14-17]. This would greatly aid fMRI and DTI studies to map functional and structural connectivity $[18,19]$.

The major challenges associated with constructing phased array coils for monkeys are the variation in head size and space constraints. Phased array technology is based on placing the multiple receive channels as close to the head as possible [20]. Therefore, the natural variation in monkey head size makes rigid coils impractical. Another major concern is the space constraints associated with awake monkey fMRI experiments (head posts, recording wells etc.). The monkeys is confined in a chair with its headpost secured to the outside of the chair [3]. This leaves very little room for RF equipment and renders bird-cage coils ineffective and makes it difficult to cover to the entire monkey brain with coils. Therefore, 
large SNR gains with phased array coils are localized to surface cortex and will not be present through the brain as in human imaging. Finally, phased array coils for awake money fMRI studies cannot interfere with the vision of the monkey.

Here, we apply phased array technology to a 4-channel phased array coil design which satisfies the criteria defined above. The coil improves the resolution and anatomical accuracy of full brain awake monkey fMRI and diffusion MRI experiments. The phased array coil is capable of adapting to different rhesus monkey head sizes (ages 4-8) [15,20]. The methodology described in this paper can be used in the development of phased array coils for other primates and small animals.

\section{MATERIALS AND METHODS}

\subsection{Single Coil-10 cm Transmit/Receive Coil}

The large majority of full brain awake monkey fMRI experiments have been performed with a single transmit/ receive coil [2-4,21]. A single transmit receive coil has the advantage of being easy to setup and is not affected by variations in monkeys head size. In order to quantitatively compare the benefits of the 4-channel phased array receive coil, we designed a $10 \mathrm{~cm}$ transmit/receive coil.

A $10 \mathrm{~cm}$ transmit/receive coil was made from flexible circuit board material (Dupont Pyralux, Durham, NC) and glued (Gougeon Corp.-G5 Adhesive Hardener and Resin Epoxy, Bay City, MI) to a thermoplastic base. The coil was formed in the shape of a 'saddle' to improve the uniformity of the field compared to a circular coil while not affecting the vision of the monkey. The conductor width was $4 \mathrm{~mm}$. The coil had seven capacitors on it and one variable capacitor (Voltronics, Denville, NJ) The coil plugged into a single preamplifier (Advanced Receiver, Burlington, CT). A schematic of the circuitry used in the transmit/receive coil is shown in Figure 1(a). The physical shape of the single transmit/receive coil and transmit only coil was the same.

\subsection{Phased Array}

\subsection{1. $10 \mathrm{~cm}$ Transmit Only Coil}

It would be ideal to use the body transmit coil of the NMR scanner in conjunction with all phased array coils because of its uniform magnetic field. The extent of the body transmit coil field induces currents in the long receive cables and causes image artifacts. Long receive cables are necessary because the preamplifiers must be located outside the magnet so that they are not affected by gradient switching. However, with a custom build 10 $\mathrm{cm}$ transmit only coil, the field of the transmitter is not large enough to reach the long cables. Thus, functional and diffusion MRI experiments are only limited in resolution by the coil properties and not other hardware considerations.

The transmit only coil was constructed in the same manner as the $10 \mathrm{~cm}$ transmit/receive coil. However, a detuning circuit was added to prevent the transmit coil from interfering with the phased array receive coil during image acquisition (see Figure 1(b)).

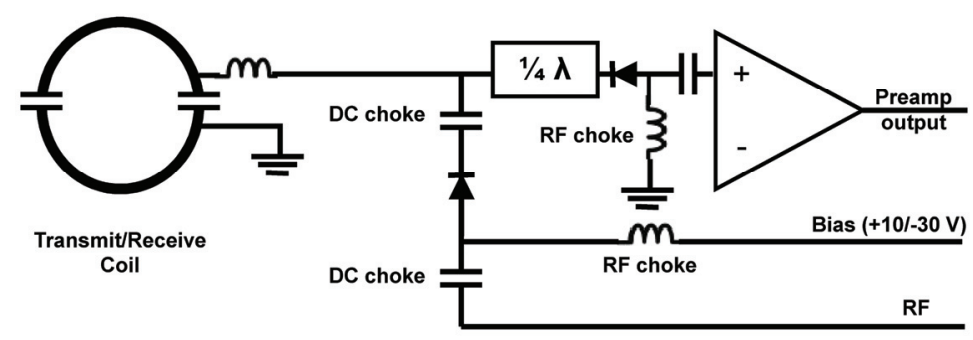

(a)

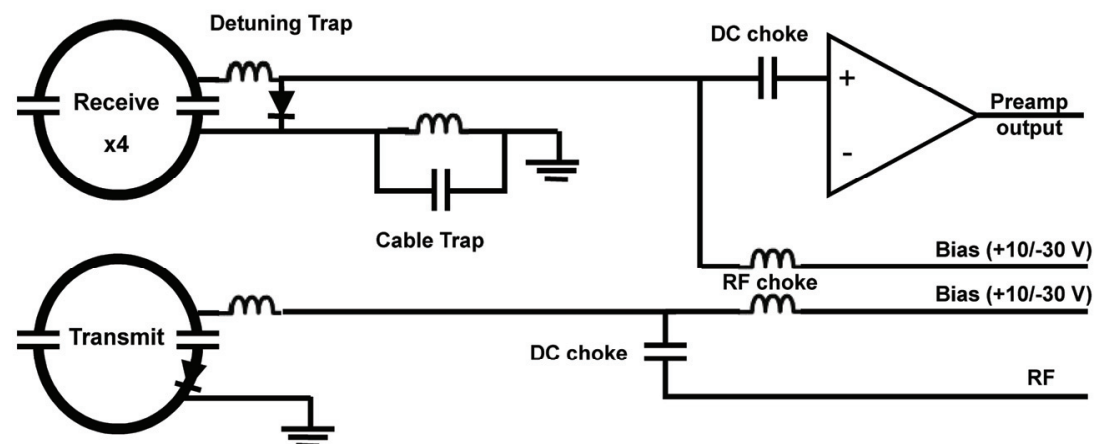

(b)

Figure 1. Circuit schematic of (a) single coil and (b) phased array coil. 


\subsubsection{4-Channel Receive Coil}

A model of a monkeys head was made from $\mathrm{T} 1$ anatomical images using 3D stereolithagraphy (Medical Modeling, Golden, CO). A monkey helmet was made from fiberglass cloth (Bondo, Atlanta, GA) and shaped to the monkey model. Three layers of fiberglass cloth were glued together using epoxy to give the monkey helmet a combination of strength and flexibility. The four receive coils were made from flexible circuit board material (Dupont Pyralux, Durham, NC) and glued to the monkey helmet (Gougeon Corp.-G5 Adhesive Hardener and Resin Epoxy, Bay City, MI). A standard capacitive bridge match and PIN diode (MA4P4002B-402; Macom, Lowell, MA) trap was used to detune the receive coils [22]. Two coils were overlapped on the left side of the helmet and two coils were overlapped on the right side. The coupling, $\mathrm{S}_{12}$, between the overlapping coils was measured $(<-20 \mathrm{~dB})$ before gluing the coil to the monkey helmet. The coupling between second nearest neighbor coils was minimized using preamplifier decoupling (see next section). Figure 1(b) shows a circuit schematic of the phased array and Figure 2 shows the orientation of the coils relative to the brain. When positioning close to the monkey, the movement of the jaw muscles do not affect the stability of the coil.

\subsection{Preamplifiers}

A preamplifier board was constructed and made portable to accommodate the different stereotactic apparatus and monkeys chairs used in awake and anesthetized MRI experiments. The preamplifier board consists of eight preamplifiers (Siemens Medical Solutions, Erlangen, Germany), and four bias lines ( $\pm 30 \mathrm{~V}$ ) to tune and detune the receive and transmit coils. Preamplifier decoupling was employed using a cable trap with semi rigid coax (Suhner UT-070 type) to suppress any residual coil coupling from next nearest neighbor interactions $[20,23]$.

\subsection{Monkey Data Acquisition}

MRI data were acquired from three juvenile male rhesus

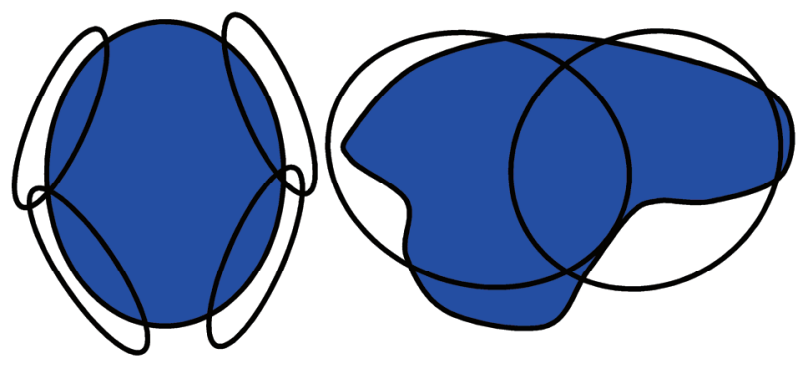

(a)

(b)

Figure 2. Schematic of the orientation of the placement of four coils relative to the brain from the (a) superior and (b) sagital view. monkeys (macaca mulattas, M1, $5.5 \mathrm{~kg}$, ID \#3704, M2, $4.9 \mathrm{~kg}$, ID \#0404, M3, $5.7 \mathrm{~kg}$, ID \#4505). The data were acquired on a Siemens Trio 3T MRI scanner located at the Athinoula A. Martinos Center for Biomedical Imaging, Massachusetts General Hospital (Charlestown, Massachusetts). All procedures conformed to Massachusetts General Hospital, Massachusetts Institute of Technology, and the National Institutes of Health guidelines for the care and use of laboratory animals (Subcommittee on Research Animal Care protocol \#2003N000338).

The monkey was placed into a magnet compatible stereotactic apparatus (Kopf Instruments, Tujunga, California) for an anaesthetized experiment to compare the performance of the single coil to the phased array coil. Anesthesia was maintained using ketamine and xylazine (induction 10 and $0.5 \mathrm{mg} / \mathrm{kg}$, i.m., maintenance with ketamine only). Local anesthetic (lidocaine cream) was applied to the ends of the ear bars and ophthalmic ointment was applied to the eyelids to minimize discomfort induced by the stereotactic apparatus. A heating pad was placed beneath the monkey to keep it warm during the scan session.

The monkey was placed in a monkey chair (Crist Instruments, Washington, DC) during awake experiments. All monkeys were implanted with a headpost (Crist Instruments, Washington, DC) which was secured to the monkey chair using two M5 peek plastic screws. Also a rail system was used to slide the monkey chair in the magnet to minimize motion. The following sequences were used to quantify the behavior of the phased array coil and single coil.

\subsection{G-Factor Maps}

Proton density gradient-echo images were acquired in order to calculate g-factor maps of the phased array coil for horizontal, coronal, and sagital slices [16,20]. Raw k-space data was obtained with TR/TE/flip $=200 \mathrm{~ms} /$ $4.14 \mathrm{~ms} / 20^{\circ}, 1.5 \mathrm{~mm}$ single slice, $128 \times 112$, and $100 \times$ $87 \mathrm{~mm}$ FOV.

\subsection{Coil Sensitivity Maps}

Proton density gradient-echo images were also acquired in order to perform a non-uniform signal normalization on the $\mathrm{T} 1$ anatomical images to improve gray and white matter contrast and aid in image registration (50 slices, $\mathrm{TR} / \mathrm{TE} / \mathrm{flip}=1190 \mathrm{~ms} / 3.72 \mathrm{~ms} / 8^{\circ}, 1.0 \mathrm{~mm}$ isotropic, 96 $\times 96$ matrix size).

\subsection{SNR Maps}

Proton density gradient-echo images (TR/TE/flip 200 $\mathrm{ms} / 3.92 \mathrm{~ms} / 20^{\circ}$, slice thickness $1 \mathrm{~mm}$, matrix $128 \times 128$, FOV $128 \mathrm{~mm}$, BW 300) were obtained in the sagital, axial, and coronal planes for SNR comparison. A noise 
reference scan was acquired by recording an image with no RF excitation. The image noise matrix, $\boldsymbol{\Psi}$, was calculated from the receive and sample noise matricies as described in [16,23] and the SNR was calculated using the equation below [24] where, $\mathbf{S}$, is the signal vector of length $n$, where $n$ is the number of coils (4 in this case).

$$
\mathrm{SNR}=\sqrt{\mathbf{S} \cdot \Psi^{-1} \cdot \mathbf{S}^{\mathrm{T}}}
$$

\section{8. fMRI}

Functional single shot echo planar images (EPI) were acquired at two different resolutions $(1.25 \mathrm{~mm}, 1.0 \mathrm{~mm}$ isotropic) to compare the anatomical accuracy of the single coil to the phased array coil.

\subsection{1. fMRI-1.0 mm BOLD}

Fifty horizontal slices were acquired using TE/TR $=26$ / $3290 \mathrm{~ms}$ (phased array coil), 26/5000 ms (single coil), $96 \times 84$ matrix, $90^{\circ}$ flip angle at $1.0 \mathrm{~mm}$ isotropic resolution. The phased array coil had a lower TR because parallel imaging [16,17,25] was employed, specifically generalized auto-calibrating partially parallel acquisitions (GRAPPA, $\mathrm{R}=2$ ) [25].

\subsection{2. fMRI-1.0 mm MION}

Fifty horizontal slices were acquired on M1, M2, and M3 using TE/TR = 24/2700 ms, $96 \times 84$ matrix, $90^{\circ}$ flip angle at $1.0 \mathrm{~mm}$ isotropic resolution using the phased array coil. The Siemens AC88 gradient insert was used (BW $=1305 \mathrm{~Hz} /$ voxel) to decrease the TR. GRAPPA with an acceleration factor of two was employed [25].

\subsection{Diffusion MRI}

Diffusion MRI was acquired at two different resolutions $(1.25 \mathrm{~mm}, 0.9 \mathrm{~mm}$ isotropic). The $1.25 \mathrm{~mm}$ data was acquired with the single coil and the $0.9 \mathrm{~mm}$ data was used to explore the practical limits of the phased array coil.

\subsubsection{Diffusion MRI-1.25 mm (Single Coil)}

The diffusion preparation used a twice-refocused spin echo [26]. Thirty horizontal slices were taken of M1 at $1.25 \mathrm{~mm}$ (0 mm skip). The in-plane resolution was $1.25 \times$ $1.25 \mathrm{~mm}$, with a matrix size of $74 \times 74(\mathrm{R}=2$ was used for the phased array coil). The sequence parameters were $\mathrm{TE} / \mathrm{TR}=85 / 8300 \mathrm{~ms}, \mathrm{~b}=700 \mathrm{~s} \mathrm{~mm}^{-2}$. The diffusion gradient sampling scheme consisted of $\mathrm{n}=60$ directions which were obtained using the electrostatic shell method [27]. Ten images with no diffusion-weighting were also obtained for a total of 70 acquisitions. The total acquisition time was $7 \mathrm{~min} 20 \mathrm{sec}$. The average of the $10 \mathrm{~b}=0$ raw images were compared for EPI distortions.

\subsubsection{Diffusion MRI-0.9 mm DTI (Phased Array Only)}

The diffusion preparation used a twice-refocused spin echo [26]. Fifty seven horizontal slices were taken of M1 at $0.9 \mathrm{~mm}$ (0 mm skip). The in-plane resolution was $0.9 \times$ $0.9 \mathrm{~mm}$, with a matrix size of $96 \times 96$. The sequence parameters were TE/TR $=93 / 10100 \mathrm{~ms}, \mathrm{~b}=700 \mathrm{~s} \mathrm{~mm}^{-2}$. The diffusion gradient sampling scheme consisted of $n=$ 60 directions which were obtained using the electrostatic shell method [27]. Ten images with no diffusion-weighting were also obtained for a total of 70 acquisitions. The total acquisition time was $11 \mathrm{~min} 48 \mathrm{sec}$. The diffusion images from five acquisitions were averaged and reconstructed using the diffusion tensor model [28].

\subsection{T1 Anatomical Images}

T1 anatomical images were acquired with an MPRAGE sequence [29] with TR/TI/TE $=1910 / 1100 / 3.06 \mathrm{~ms}, \alpha=$ $8^{\circ}, 0.65 \mathrm{~mm}$ isotropic resolution, total acquisition time: 6 min $35 \mathrm{sec}$. The functional (EPI), proton density and GRE images were registered to the T1 images for comparison between the single and phase array coils.

\subsection{Image Registration and Visualization}

Images were registered using the flirt command (rigid registration, 6 degrees of freedom) in the FSL toolbox (http://www.fmrib.ox.ac.uk). All visualization post-processing was performed using custom software written in Matlab (Version 6.5.1.199709 (R13) Service Pack 1). DTI reconstructions were visualized with custom software written in $\mathrm{C}++$ and VTK (Version 4.2) (http://public. kitware.com/VTK). The tensors were visualized as color-coded rectangloids.

\section{RESULTS}

\subsection{Coil Properties}

The coupling between the coils was measured using an $\mathrm{S}_{12}$ measurement on a network analyzer. The two sets of overlapping coils had $<-20 \mathrm{~dB}$ decoupling between them. Next nearest neighbor coupling was $<-10 \mathrm{~dB}$. In addition, the preamp decoupling added $-20 \mathrm{~dB}$ of decoupling. Therefore, all coils had $<-30 \mathrm{~dB}$ of decoupling between them ensuring each coil behaved as a single element in the tuned state. PIN diode detuning achieved $>35 \mathrm{~dB}$ of isolation between the tuned and detuned states.

\subsection{SNR}

The SNR in the four individual coils of the phased array is shown in Figure 3 and was calculated using (1).

Figure 4 compares the SNR of the single coil to the phased array coil. The phased array coil has higher SNR in surface cortex but has a less uniform profile. Table 1 presents the relative SNR of the phased array coil to the single coil in regions relevant to fMRI studies. 


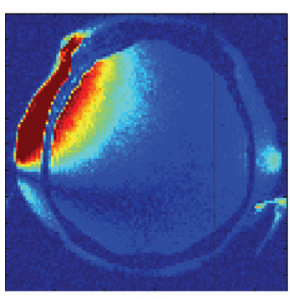

(a)

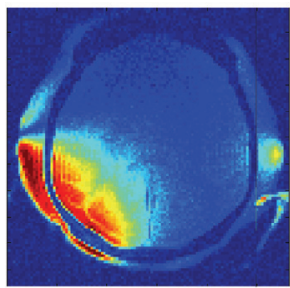

(c)

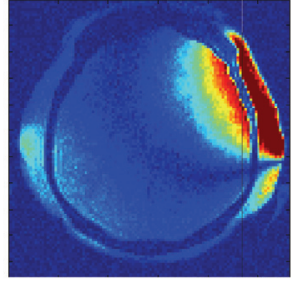

(b)

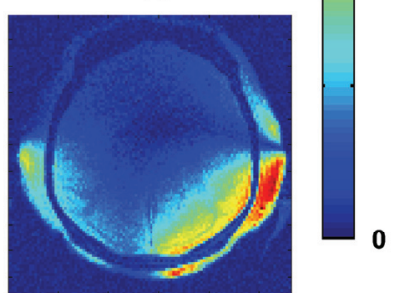

(d)
Figure 3. (a)-(d) SNR of individual coils.

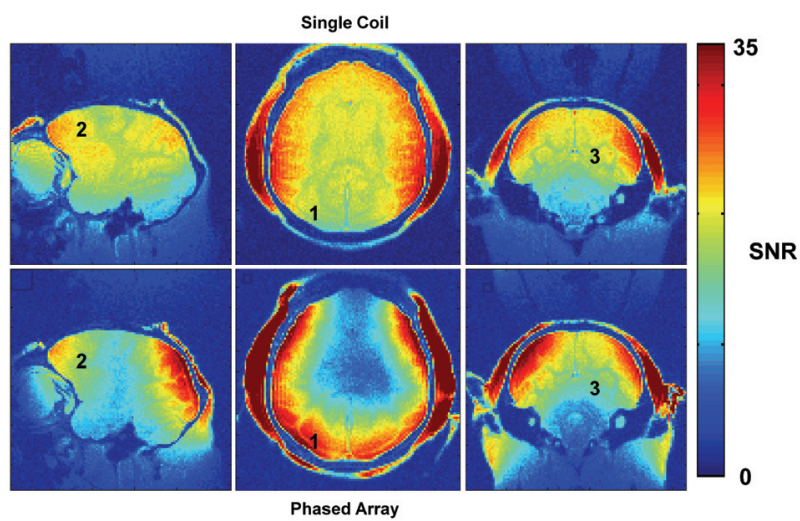

Figure 4. (Top) SNR of single coil and (Bottom) SNR of phased array coil. The SNR of the phased array coil is higher than the single coil in surface cortex although the single coil has a more uniform SNR profile.

Table 1. Relative SNR in the phased array coil relative to the single coil in primary visual cortex (V1), the frontal eye fields (FEF), and the lateral geniculate nucleus (LGN) in the left and right hemispheres (LH, RH).

\begin{tabular}{ccc}
\hline \multirow{2}{*}{ Region } & \multicolumn{2}{c}{ SNR $_{\mathbf{P A}_{\mathrm{A}}} / \mathbf{S N R}_{\mathrm{SC}}$} \\
\cline { 2 - 3 } & $\mathbf{L H}$ & $\mathbf{R H}$ \\
\hline V1 & 2.10 & 2.03 \\
FEF & 0.76 & 0.74 \\
LGN & 0.80 & 0.78 \\
\hline
\end{tabular}

\section{3. g-factor}

An important property of a phased array coil is its g-factor map. The inverse g-factor map quantifies how much SNR is lost in the brain during a parallel acquisition $(\mathrm{R}>$
1) compared to an image with no acceleration, $R=1$. The parallel imaging capabilities of a 4 channel coil limit the acceleration factor to two, (i.e., $\mathrm{R}=2$ ) [16]. Figure 5 presents the inverse g-factor maps for the phased array coil for an acceleration factor of two in the different phase encode directions. Less than $15 \%$ of the SNR is lost during an acceleration factor of two in $85 \%$ of the brain. The maximum g-factor of 1.35 (Figure 5(a)) occurs in the horizontal slice with left-right phase encoding.

\section{4. fMRI}

Functional EPI (BOLD) was acquired at $1.0 \mathrm{~mm}$ isotropic resolution. The TR of the phased array coil was much shorter (3290 ms) compared to that of the single coil (5000) because parallel imaging (GRAPPA, $\mathrm{R}=2$ ) was employed. Figure 6 presents EPI at $1.0 \mathrm{~mm}$ isotropic for the single and phased array coil. The benefit of parallel imaging with regards to EPI distortions is apparent around the edge of the brain. A T1 image is presented for reference.

Figure 7 presents functional EPI (MION) of M1, M2, and M3. The images were acquired with the AC88 gradient insert (Siemens, Erlangen, Germany). The SNR profiles in the brain are relatively consistent between the monkeys despite the natural variation in head size. M1 has the largest head and thus the signal in the middle of the brain is the lowest compared to M2 and M3.

\subsection{Diffusion MRI}

Figure 8 presents the average of 10 T2 images $(b=0)$ from a DTI scan from a single coil $(1.25 \mathrm{~mm})$ and phased array coil $(\mathrm{R}=2,0.9 \mathrm{~mm})$. The severe EPI distortions in

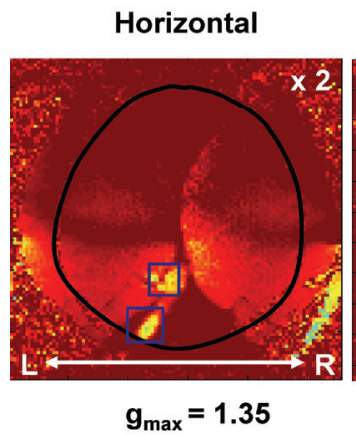

(a)

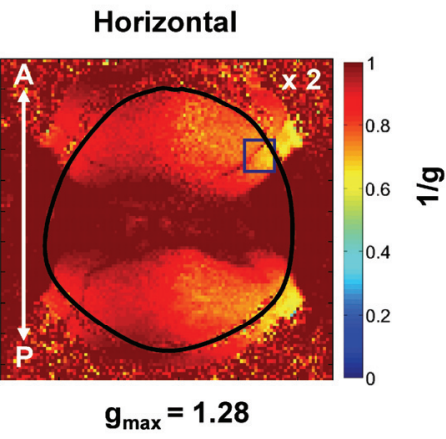

(b)
Figure 5. 1/g-factor maps for the (a) left-right horizontal and the (b) anterior-posterior horizontal phase encode directions for two-fold GRAPPA acceleration, $\mathrm{R}=2$. The blue boxes indicate where the maximum $g$-factor in the slice is located. In $85 \%$ of the brain, the SNR decrease is less than $15 \%$ for two-fold GRAPPA acceleration. The average SNR decrease in the brain is $\sim 50 \%$ for $\mathrm{R}=3$ (data not shown). The monkey's brain is outlined in black. 


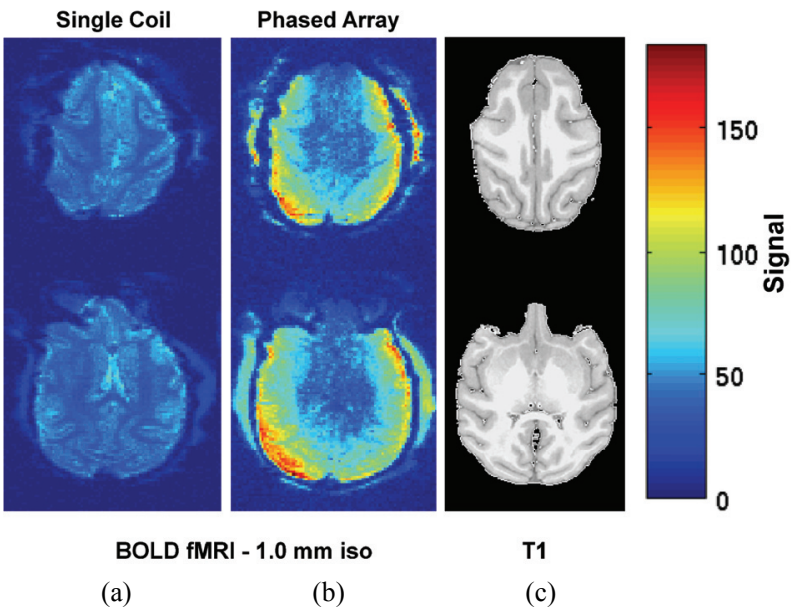

Figure 6. SNR of (a) single and (b) phased array functional EPI at $1 \mathrm{~mm}$ isotropic resolution. Both images are on the same scale. The phased array coil images where taken with a GRAPPA acceleration factor of 2 . The phased array image does not posses the EPI distortions of the single coils are more accuratley represents the $\mathrm{T} 1$ image in panel (c).

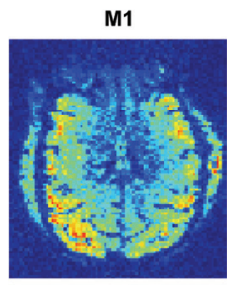

(a)

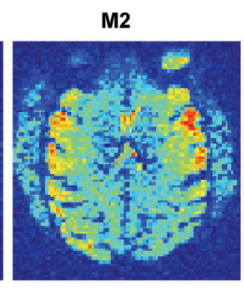

MION fMRI $-1.0 \mathrm{~mm}$ iso

(b) (c)

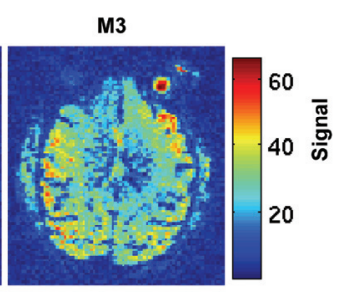

Figure 7. Functional EPI (with MION) acquired with the phased array coil for (a) M1, (b) M2, and (c) M3 at $1.0 \mathrm{~mm}$ isotropic resolution, TE/TR $=24 / 2700 \mathrm{~ms}$. M1 has a larger brain than M2 and M3 resulting in lower SNR in the middle of the brain. However the SNR in all monkeys is sufficient $(>25)$ to measure the MION response throughout cortex.
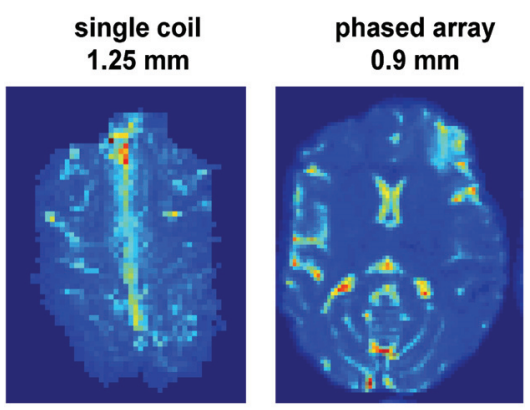

DTI T2-weighted image $(b=0)$

(a)

(b)

(c)
Figure 8. (a) Single coil (1.25 mm) and (b) phased array (0.9 $\mathrm{mm}) \mathrm{b}=0 \mathrm{~T} 2$-weighted images $(\mathrm{R}=2)$ for a DTI scan. The severe EPI distortions in the single coil image make registration very difficult with a (c) T1 image compared the phased array coil image. the single coil image make registration very difficult with a T1 image. Figure 9 depicts the DTI reconstruction of a $0.9 \mathrm{~mm}$ isotropic acquisition. The tensor map is overlayed on a $\mathrm{T} 1$ with a rigid registration algorithm.

The phased array coil provides a much more accurate anatomical image such that the rigid registration preserves the tensor maps. ADC and FA maps calculated from the tensors are presented in panels (b) and (c). The phased array coil is capable of resolving the ADC and FA throughout the white matter in the brain.

\section{DISCUSSION}

The SNR benefit of the phased array coil compared to the single coil in surface visual cortex, make it ideal for awake monkey fMRI retinotopic studies (Figure 3, Figure 4, Table 1). In addition the benefit of using the phased array coil with parallel imaging improves the resolution of awake monkey fMRI experiments at $3 \mathrm{~T}$ by a factor of 2 (Figure 6) (1.25 $\mathrm{mm}$ to $1.0 \mathrm{~mm}$ isotropic) and diffusion MRI experiments by a factor of 4 (Figure 8 and Figure 9) (1.5 $\mathrm{mm}$ to $0.9 \mathrm{~mm}$ isotropic). The preservation of the anatomical integrity of the fMRI and diffusion images results in accurate registration with $\mathrm{T} 1$ images with a rigid registration algorithm. Thus, functional and diffusion tensor maps can be visualized on a T1 image making tractography more accurate. During parallel imaging, an SNR loss of less than $15 \%$ is present over $85 \%$ of the brain with a maximum loss of $26 \%$, GRAPPA $=2$ (Figure 5). The SNR decrease in surface cortex due to parallel imaging is more than compensated by the coils higher SNR in that region (i.e., $26 \%$ decrease during parallel imaging compared to $100 \%$ boost compared to single coil). The coil does not interfere with the eyes of the monkey or with the headpost because all of the coils are on the side of the head (Figure 2). This allows eye tracking to be performed during fMRI experiments. In addition, the flexibility of the fiberglass helmet allows the coil to easily adapt to different monkeys (Figure 7).

Though four coils were used in this phased array, future phased arrays with more coils could be constructed using the same techniques described in this paper. The general methodology of making a monkey mold from $\mathrm{T} 1$ anatomical images and then constructing a fiberglass helmet, can be applied to primates and other animals. The application of the coil should be taken into account when developing a phased array coil. For example, in fMRI studies only interested in retinotopy, it may be more suitable to use more surface elements and sacrifice the coils performance in deep cortical structures. Also, for T1 anatomical studies of rhesus monkeys, a headpost is not necessary. This would allow one to put a coil on top of the brain making the phased array coil superior to the 


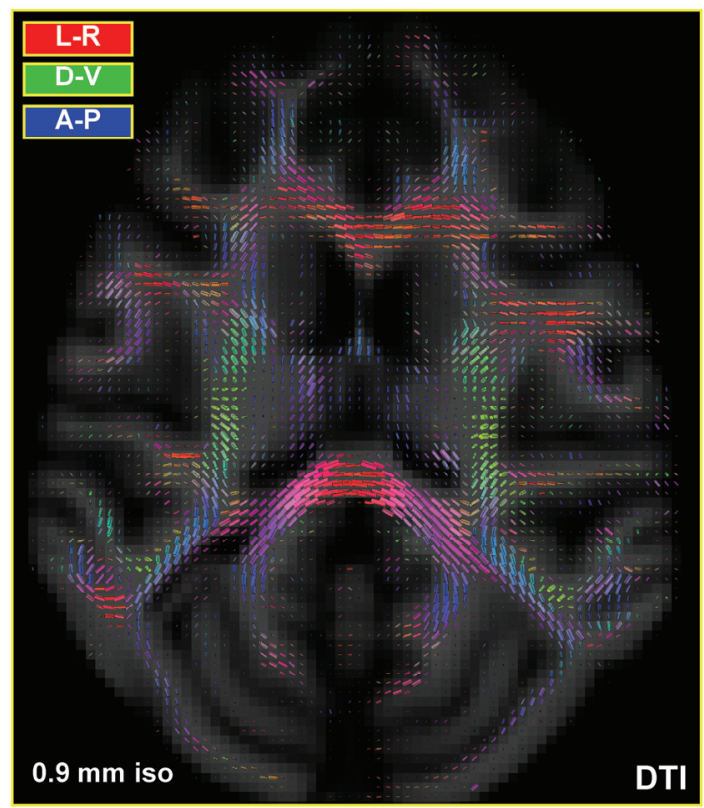

(a)

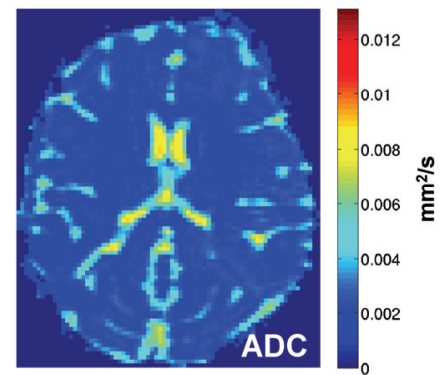

(b)

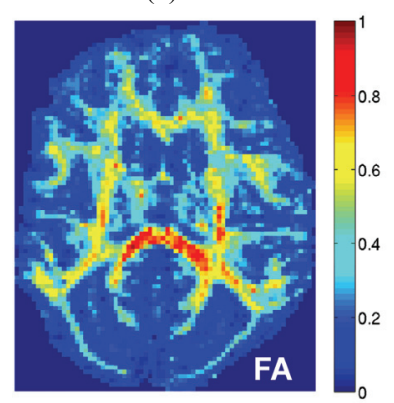

(c)

Figure 9. (a) DTI, (b) ADC, and (c) FA maps acquired with the phased array coil at $0.9 \mathrm{~mm}$ isotropic resolution. The phased array coil resolves the ADC and FA throughout the brain.

single coil throughout the brain.

\section{CONCLUSIONS}

A 3 Tesla 4-channel phased array coil was developed to improve the resolution and anatomical accuracy (i.e., reduction of EPI distortions) of awake monkey fMRI and diffusion MRI experiments. The novel aspect of the phased array coil is that can adapt to different rhesus monkey head sizes (ages 4-8) and fits in the limited space provided by monkey stereotactic equipment. The phased array coil had a SNR benefit of $\sim 2$ on surface cortex. The phased array coil allowed parallel imaging to be employed reducing EPI distortions. It improved the resolution of fMRI studies by a factor of $2(1.25 \mathrm{~mm}$ to 1 $\mathrm{mm}$ isotropic). When applied to diffusion MRI studies, the phased array coil improved the resolution of diffusion MRI images by a factor of $4(1.5 \mathrm{~mm}$ to $0.9 \mathrm{~mm}$ isotropic) compared to single coil studies. The flexibility of the design allows the coil to adapt to monkeys with varying head sizes.

\section{ACKNOWLEDGEMENTS}

This work would not have been possible without the guidance of Graham Wiggins and Larry Wald. The author is also grateful to Wim Vanduffel, Hauke Kolster, John T. Arsenault, Leeland Ekstrom, Bechir Jarraya, Andreas Potthast, Simon Sigalovsky, and Helen Deng for their assistance with this project. This work was supported by IUAP 5/04, EF/05/014, FWO G151.04, HFSPO RGY0014/2002-C, GSKE, NINDS NS46532, NCRR RR14075, NCI CA09502, NCI 5T32CA09502,
GlaxoSmithKline, the Athinoula A. Martinos Foundation, the Mental Illness and Neuroscience Discovery (MIND) Institute, and the National Alliance for Medical Image Computing (NAMIC) (NIBIB EB05149) which is funded through the NIH Roadmap for Medical Research.

\section{REFERENCES}

[1] Ekstrom, L.B., Roelfsema, P.R., Arsenault, J.T., Bonmassar, G. and Vanduffel, W. (2008) Bottom-up dependent gating of frontal signals in early visual cortex. Science, 321, 414-417.

[2] Sasaki, Y., Rajimehr, R., Kim, B.W., Ekstrom, L.B., Vanduffel, W. and Tootell, R.B. (2006) The radial bias: A different slant on visual orientation sensitivity in human and nonhuman primates. Neuron, 51, 661-670.

[3] Vanduffel, W., Fize, D., Mandeville, J.B., Nelissen, K., Van Hecke, P., Rosen, B.R., Tootell, R.B. and Orban, G.A. (2001) Visual motion processing investigated using contrast agent-enhanced fMRI in awaking behaving monkeys. Neuron, 32, 565-577.

[4] Tsao, D.Y., Vanduffel, W., Sasaki, Y., Fize, D., Knutsen, T.A., Mandeville, J.B., Wald, L.L., Dale, A.M., Rosen, B.R., Van Essen, D.C., Livingstone, M.S., Orban, G.A. and Tootell, R.B. (2003) Stereopsis activates V3A and caudal intraparietal areas in macaques and humans. Neuron, 39, 555-568.

[5] Khachaturian, M.H., Arsenault, J., Ekstrom, L.B., Tuch, D.S. and Vanduffel, W. (2008) Focal reversible deactivation of cerebral metabolism affects water diffusion. Magnetic Resonance in Medicine, 60, 1178-1189.

[6] Behrens, T.E., Johansen-Berg, H., Woolrich, M.W., Smith, S.M., Wheeler-Kingshott, C.A., Boulby, P.A., 
Barker, G.J., Sillery, E.L., Sheehan, K., Ciccarelli, O., Thompson, A.J., Brady, J.M. and Matthews, P.M. (2003) Non-invasive mapping of connections between human thalamus and cortex using diffusion imaging. Nature Neuroscience, 6, 750-757.

[7] Gerig, G., Gouttard, S. and Corouge, I. (2004) Analysis of brain white matter via fiber tract modeling. Conference Proceedings - IEEE Engineering in Medicine and Biology Society, 6, 4421-4424.

[8] Price, G., Cercignani, M., Parker, G.J., Altmann, D.R., Barnes, T.R., Barker, G.J., Joyce, E.M. and Ron, M.A. (2007) Abnormal brain connectivity in first-episode psychosis: A diffusion MRI tractography study of the corpus callosum. Neuroimage, 35, 458-466.

[9] Yamamoto, A., Miki, Y., Urayama, S., Fushimi, Y., Okada, T., Hanakawa, T., Fukuyama, H. and Togashi, K. (2007) Diffusion tensor fiber tractography of the optic radiation: analysis with 6-, 12-, 40-, and 81-directional motion-probing gradients, a preliminary study. American Journal of Neuroradiology, 28, 92-96.

[10] Tuch, D., Wisco, J., Khachaturian, M., Ekstrom, L., Kotter, R. and Vanduffel, W. (2005) Q-ball imaging of macaque white matter architecture. Philosophical Transactions of the Royal Society B: Biological Sciences, 360(1457), 869-879.

[11] Khachaturian, M.H., Wisco, J.J. and Tuch, D.S. (2007) Boosting the sampling efficiency of q-Ball imaging using multiple wavevector fusion. Magnetic Resonance in Medicine, 57, 289-296.

[12] Tuch, D.S. (2004) Q-ball imaging. Magnetic Resonance in Medicine, 52, 1358-1372.

[13] Wedeen, V., Reese, T.G., Tuch, D.S., Weigel, M.R., Dou, J.G., Weiskoff, R.M. and Chessler, D. (2000) Mapping fiber orientation spectra in cerebral white matter with fourier-transform diffusion MRI. Proceedings of International Society Magnetic Resonance in Medicine, Denver, 82.

[14] Hayes, C.E, Hattes, N. and Roemer, P.B. (1991) Volume imaging with MR phased arrays. Magnetic Resonance in Medicine, 18, 309-319.

[15] Wright, S.M. and Wald, L.L. (1997) Theory and application of array coils in MR spectroscopy. NMR in Biomedicine, 10, 394-410.

[16] Pruessmann, K.P., Weiger, M., Scheidegger, M.B., Boesige, P. (1999) SENSE: sensitivity encoding for fast MRI. Magnetic Resonance in Medicine, 42, 952-962.

[17] Sodickson, D.K., Griswold, M.A. and Jakob, P.M. (1999)
Smash imaging. Magnetic Resonance Imaging Clinics of North America, 7, 237-254.

[18] Jaermann, T., Crelier G, Pruessmann KP, Golay X, Netsch T, van Muiswinkel AM, Mori S, van Zijl PC, Valavanis A, Kollias S, Boesiger P. SENSE-DTI at $3 \mathrm{~T}$. Magn Reson Med. 2004; 51, 230-236.

[19] Jaermann, T., Pruessmann, K.P., Valavanis, A., Kollias, S. and Boesiger, P. (2006) Influence of SENSE on image properties in high-resolution single-shot echo-planar DTI. Magnetic Resonance in Medicine, 55, 335-342.

[20] Wiggins, G.C., Triantafyllou, C., Potthast, A., Reykowski, A., Nittka, M. and Wald, L.L. (2006) 32-channel 3 Tesla receive-only phased-array head coil with soccer-ball element geometry. Magnetic Resonance in Medicine, 56, 216-223.

[21] Tootell, R.B., Tsao, D. and Vanduffel, W. (2003) Neuroimaging weighs in: humans meet macaques in "primate" visual cortex. Journal of Neuroscience, 23, 3981-3989.

[22] Hardy, C.J., Edelstein, W.A. and Mueller, O.M. (1986) Surface-coil T1 images. Magn Reson Med., 3, 935-40.

[23] Roemer PB, Edelstein WA, Hayes CE, Souza SP, Mueller OM. (1990) The NMR phased array. Magnetic Resonance in Medicine, 16, 192-225.

[24] Constantinides, C.D., Westgate, C.R., O'Dell, W.G., Zerhouni, E.A. and McVeigh, E.R. (1995) A phased array coil for human cardiac imaging. Magnetic Resonance in Medicine, 34, 92-98.

[25] Griswold, M.A., Jakob, P.M., Heidemann, R.M., Nittka, M., Jellus, V., Wang, J., Kiefer, B. and Haase, A. (2002) Generalized autocalibrating partially parallel acquisitions (GRAPPA). Magnetic Resonance in Medicine, 47, 12021210.

[26] Reese, T.G., Heid, O., Weisskoff, R.M. and Wedeen, V.J. (2003) Reduction of eddy-current-induced distortion in diffusion MRI using a twice-refocused spin echo. Magnetic Resonance in Medicine, 49, 177-182.

[27] Jones, D.K., Horsfield, M.A. and Simmons, A. (1999) Optimal strategies for measuring diffusion in anisotropic systems by magnetic resonance imaging. Magnetic Resonance in Medicine, 42, 515-525.

[28] Basser, P.J., Mattiello, J., LeBihan, D. (1994) MR diffusion tensor spectroscopy and imaging. Biophysics Journal, 66, 259-267.

[29] Mugler, J.P. and Brookeman, J.R. (1990) Three-dimensional magnetization-prepared rapid gradient-echo imaging (3D MP RAGE). Magnetic Resonance in Medicine, 15, 152-157. 